\title{
Implicit time discretization of the mean curvature flow with a discontinuous forcing term
}

\author{
A. Chambolle ${ }^{\dagger}$ \\ CMAP, CNRS UMR 7641, École Polytechnique, 91128 Palaiseau Cedex, France \\ M. NovagA \\ Dipartimento di Matematica, Università di Pisa, Largo B. Pontecorvo 5, 56127 Pisa, Italy
}

[Received 28 November 2006 and in revised form 16 April 2008]

\begin{abstract}
We consider an implicit time discretization for the motion of a hypersurface driven by its anisotropic mean curvature. We prove some convergence results of the scheme under very general assumptions on the forcing term, which include in particular the case of a typical path of the Brownian motion. We compare this limit with other available solutions, whenever they are defined. As a by-product of the analysis, we also provide a simple proof of the coincidence of the limit flow with the regular evolutions, defined for small times, in the case of a regular forcing term.
\end{abstract}

\section{Introduction}

Mean curvature flow has attracted a lot of attention in the past few years. Although it is one of the simplest evolutions of hypersurfaces of $\mathbb{R}^{n}$, in its analysis many difficult issues arise, mainly related to the formation of singularities, which sometimes lead to changes of the topology. To deal with this phenomenon, several notions of weak solutions have been proposed, such as (to mention but a few) the varifold theory of Brakke [10], the level-set solution defined through viscosity theory [18, 19, [15], the minimal barrier method of De Giorgi [16], the limit of a reaction-diffusion equations [14, 21] and the minimizing movements method [1, 25, 2], which corresponds to an implicit time-discrete scheme.

Each of these methods has different features and presents advantages and disadvantages. In particular, the level-set method always provides a unique solution, globally defined in time in the class of compact subsets of $\mathbb{R}^{n}$, but it is often very difficult to prove that such a solution is a regular hypersurface. There are even some singular situations in which this solution becomes a compact set with nonempty interior, showing the so-called fattening phenomenon. The minimal barrier method is a geometric counterpart of the level-set method and produces essentially the same solution [6].

On the contrary, the minimizing movements method produces a solution, called the flat flow, which may be nonunique but is always a (possibly nonsmooth) hypersurface. One of the difficulties in this approach is to show that the solution coincides with the classical smooth solution, whenever the latter exists, a property which is very easy to prove in the context of level-set viscosity solutions. One faces similar difficulties in proving that the flat flow is always contained in the level-set solution.

In this paper we study the (anisotropic) mean curvature flow with a possibly discontinuous driving force, by adapting the minimizing movements method, which has been originally developed

\footnotetext{
†E-mail: antonin.chambolle@polytechnique.fr
}

"E-mail: novaga@dm.unipi.it 
without any forcing term. More precisely, we consider the evolution $E(t)$ of a set whose boundary is driven by the velocity

$$
V(x, t)=-\left(\kappa_{\phi}(x, t)+g(x, t)\right) n_{\phi}(x, t)
$$

for any $x \in \partial E(t)$, where $\kappa_{\phi}(x, t)$ and $n_{\phi}(x, t)$ are respectively the $\phi$-curvature and $\phi$-normal to $\partial E(t)$ at $x$ (see Section 2 for the precise definitions, and [9] for a general introduction on curvatures in Finsler geometry).

The purpose of this paper is twofold:

1. We extend the method of minimizing movements (and the proofs of consistency in [13]) to evolutions with a driving force, providing simple proofs of the coincidence with regular solutions and the inclusion in the level-set solution (see Cor.4.6 and Props. 4.8, 4.9).

2. Our approach applies to the case where the forcing term is discontinuous. One important example is a forcing term which is the time derivative of a Hölder continuous function $G(t)$, e.g., a typical path of the Brownian motion $d W / d t$. It also covers the case of spatially correlated Brownian motion, typically of the form $g(x, t)=d W / d t(t)+g^{0}(x, t)$ where $g^{0}$ is Lipschitz-continuous in $x$ and continuous in $t$ (see (18) for a precise formulation). A theory yielding existence and uniqueness for such evolutions, based on a level-set formulation in the framework of viscosity theory, has recently been developed in [23, 24], and a corresponding theory in the framework of minimal barriers, valid only for $x$-independent forcing terms, has been proposed in [17]. We also refer to [26, 27] for a similar approach to a related problem, which still uses an implicit time discretization procedure.

We do not address in the present paper the issue of continuity in time (in a suitable topology) of the limit flat flow, even if we prove some weaker continuity results with respect to the Hausdorff distance (see Props. 4.3, 4.4 and Remark 4.5).

The plan of this paper is as follows. In Section 2, we define the appropriate notion of sub- and superflow associated to the evolution equation (1), and we recall the definition of maximal and minimal barriers in the sense of De Giorgi. These allow one to define generalized evolutions, which are essentially equivalent to the evolutions defined in terms of level sets of viscosity solutions, when the forcing term is regular enough.

Then our implicit time-discretization scheme is defined in Section 3, and we show that it is consistent with our sub- and superflows (Thm. 3.3). As a corollary, we obtain a comparison results for sub- and superflows, which follows from the monotonicity of our scheme.

This consistency result is used in Section 4 to study the convergence of our time-discrete scheme, as the time step goes to zero. We define a notion of weak solution starting from an initial surface $\partial E$ (given by $\Gamma(t)=E^{*}(t) \backslash E_{*}(t)$, where $E_{*}(t) \subset E^{*}(t)$ are two evolving sets with $E_{*}(0)=\operatorname{int}(E)$, $E^{*}(0)=\bar{E}$ ), which coincides with the barrier solution as long as the latter is unique (Cor. 4.6.). Under additional assumptions on the evolution law, we deduce that it is contained in the zero levelset of the viscosity solution (Prop. 4.8). This is also true for a particular class of stochastic evolutions (Prop. 4.9.

In Sections 4.2 4.3, under some further assumption on the forcing term (which still allows a stochastic forcing), we build a level-set evolution $u(t)$ starting from any bounded, uniformly continuous function $u_{0}$. In particular, for all initial data $\left\{u_{0}=s\right\}$ but a countable number, it shows that we can define a generalized flow $\{u(t)=s\}$ which remains a continuous hypersurface (with possible singularities). We do not prove that it is unique, though. If the forcing is regular, $u(t)$ is the 
same as the unique viscosity solution of the geometric equation associated to the flow. In general, we expect it to coincide with the solution defined by Lions and Souganidis in [23, 24].

\section{Preliminary definitions and results}

Let $\phi: \mathbb{R}^{N} \rightarrow \mathbb{R}$ be a norm on $\mathbb{R}^{N}$ (that is, an even, convex, one-homogeneous function) such that $\phi \in C^{2}\left(\mathbb{R}^{N} \backslash\{0\}\right.$ ) (we shall simply say that $\phi$ is smooth) and $\nabla^{2}\left(\phi^{2}\right) \geqslant c$ Id for some $c>0$, so that $\phi$ is uniformly convex or elliptic. Most of our results could be extended to more general norms (or even possibly noneven convex one-homogeneous functions), but the proof of the consistency theorem 3.3. in the form presented here, needs such a regularity. Moreover, providing a clear and sound definition of a sub/superflow, as in Definition 2.1 below, is more difficult if the anisotropy is only Lipschitz-continuous, or nonelliptic.

Let $\phi^{\circ}$ be the polar norm, that is, $\phi^{\circ}(\xi):=\sup _{\phi(\eta) \leqslant 1} \xi \cdot \eta$ for all $\xi \in \mathbb{R}^{N}$. It turns out that $\phi^{\circ}$ is smooth and elliptic. The couple $\left(\phi, \phi^{\circ}\right)$ will be referred as the anisotropy. A ball of radius $r>0$ centred at $x_{0} \in \mathbb{R}^{N}$ for the norm $\phi$, i.e., the set $W_{\phi}\left(x_{0}, r\right):=\left\{\phi\left(x-x_{0}\right) \leqslant \rho\right\}$, will be called a Wulff shape (we set for simplicity $W_{\phi}:=W_{\phi}(0,1)$ ).

When $E, F \subset \mathbb{R}^{N}$, we denote by $\operatorname{dist}_{\phi}(E, F)$ the distance between $E$ and $F$ with respect to $\phi$ :

$$
\operatorname{dist}_{\phi}(E, F):=\inf _{x \in E, y \in F} \phi(x-y) .
$$

Given a set $E \subset \mathbb{R}^{N}$, we also define $d_{E}(x)$, the signed distance function to $\partial E$ (with respect to the norm $\phi$ ), by

$$
d_{E}(x):=\inf _{y \in E} \phi(x-y)-\inf _{y \in \mathbb{R}^{N} \backslash E} \phi(y-x) .
$$

We let $n_{\phi}(x):=\nabla \phi^{\circ}\left(\nabla d_{E}(x)\right)$ and $\kappa_{\phi}(x):=\operatorname{div} n_{\phi}(x)$ be respectively the $\phi$-normal and $\phi$ curvature of $\partial E$ at $x$. Notice that if $\partial E$ is of class $C^{2}$, then the functions $n_{\phi}$ and $\kappa_{\phi}$ are defined and continuous in an open neighbourhood of $\partial E$. We refer to [9] for a general introduction to the anisotropic curvature flow.

We say that $E$ satisfies an interior (resp. exterior) $\varepsilon W_{\phi}$-condition, $\varepsilon>0$, if $E=\left\{d_{E}<-\varepsilon\right\}+$ $\varepsilon W_{\phi}$ (resp. $\mathbb{R}^{N} \backslash E=\left\{d_{E}>\varepsilon\right\}+\varepsilon W_{\phi}$ ), which is equivalent to requiring that at each point of $\partial E$, there is a Wulff shape of radius $\varepsilon$ inside $E$ (resp., outside $E$ ) that is tangent to $\partial E$ at $x$.

\subsection{Evolution law}

Definition 2.1 Let $E(t) \subset \mathbb{R}^{N}, t \in\left[t_{0}, t_{1}\right]$, be closed sets. We say that $E(t)$ is a superflow of (1) if there exist a bounded open set $A \subset \mathbb{R}^{N}$ with $\bigcup_{t_{0} \leqslant t \leqslant t_{1}} \partial E(t) \times\{t\} \subset A \times\left[t_{0}, t_{1}\right]$ and $\delta>0$ such that $d(x, t)=d_{E(t)}(x) \in C^{0}\left(\left[t_{0}, t_{1}\right] ; C^{2}(A)\right)$, and

$$
d(x, s)-d(x, t) \geqslant \int_{t}^{s} \operatorname{div} \nabla \phi^{\circ}(\nabla d)(x, \tau) \mathrm{d} \tau+G(x, s)-G(x, t)+\delta(s-t)
$$

for a.e. $x \in A$ and any $t, s$ with $t_{0} \leqslant t \leqslant s \leqslant t_{1}$, where $G(x, t):=\int_{0}^{t} g(x, s) \mathrm{d} s$.

We say that $E(t)$ is a subflow whenever there exist $A \subset \mathbb{R}^{N}$ as above and $\delta<0$ such that the reverse inequality holds in $(2)$.

We denote by $\mathcal{F}^{+}$(resp. $\mathcal{F}^{-}$) the family of all superflows (resp. subflows) of (1). 
We observe that if $g$ is continuous in $(x, t)$, and $d$ is $C^{1}$ in $t$, condition (2) is equivalent to

$$
\frac{\partial d}{\partial t}>\operatorname{div} \nabla \phi^{\circ}(\nabla d)+g \quad \text { in } A \times\left[t_{0}, t_{1}\right] .
$$

On the other hand, Definition 2.1 still makes sense if the driving term is the "time-derivative" of a function $G \in C^{0}\left(\left[t_{0}, t_{1}\right] ; L^{\infty}(A)\right)$, even when $G$ is nondifferentiable with respect to $t$.

\subsection{Barriers}

We recall the definition of minimal and maximal barrier in the sense of De Giorgi. We refer to [8, 5, 7] for a more general introduction to this topic.

DEFINITION 2.2 We say that a function $\Phi:\left[t_{0},+\infty\right) \rightarrow \mathcal{P}\left(\mathbb{R}^{N}\right)\left(\mathcal{P}\left(\mathbb{R}^{N}\right)\right.$ is the set of all subsets of $\mathbb{R}^{N}$ ) is a barrier with respect to $\mathcal{F}^{+}$if for any $\Sigma(t) \in \mathcal{F}^{+}$with $t \in[a, b] \subset\left[t_{0},+\infty\right), \Sigma(a) \subseteq$ $\Phi(a)$ implies $\Sigma(b) \subseteq \Phi(b)$.

Similarly, we say that $\Phi$ is a barrier with respect to $\mathcal{F}^{-}$if for any $\Sigma(t) \in \mathcal{F}^{-}$with $t \in[a, b] \subset$ $\left[t_{0},+\infty\right), \Sigma(a) \supseteq \Phi(a)$ implies $\Sigma(b) \supseteq \Phi(b)$.

In the following we denote by $\mathcal{B}_{t_{0}}^{ \pm}$the class of all barriers with respect to $\mathcal{F}^{ \pm}$, defined on $\left[t_{0},+\infty\right)$.

Definition 2.3 Let $E \subseteq \mathbb{R}^{N}, t_{0} \in \mathbb{R}$. The minimal barrier $\mathcal{M}\left(E, t_{0}\right):\left[t_{0},+\infty\right) \rightarrow \mathcal{P}\left(\mathbb{R}^{N}\right)$ starting from $E$ at time $t_{0}$ is defined as

$$
\mathcal{M}\left(E, t_{0}\right)(t):=\bigcap\left\{\Phi(t): \Phi \in \mathcal{B}_{t_{0}}^{+}, \Phi\left(t_{0}\right) \supseteq E\right\} .
$$

We define the maximal barrier $\mathcal{N}\left(E, t_{0}\right):\left[t_{0},+\infty\right) \rightarrow \mathcal{P}\left(\mathbb{R}^{N}\right)$ starting from $E$ at time $t_{0}$ as

$$
\mathcal{N}\left(E, t_{0}\right)(t):=\bigcup\left\{\Phi(t): \Phi \in \mathcal{B}_{t_{0}}^{-}, \Phi\left(t_{0}\right) \subseteq E\right\}
$$

We also define the upper and lower regularized barriers as

$$
\begin{aligned}
\mathcal{M}_{*}\left(E, t_{0}\right)(t):=\bigcup_{\rho>0} \mathcal{M}\left(E_{\rho}^{-}, t_{0}\right)(t), \quad \mathcal{M}^{*}\left(E, t_{0}\right)(t):=\bigcap_{\rho>0} \mathcal{M}\left(E_{\rho}^{+}, t_{0}\right)(t), \\
\mathcal{N}_{*}\left(E, t_{0}\right)(t):=\bigcup_{\rho>0} \mathcal{N}\left(E_{\rho}^{-}, t_{0}\right)(t), \quad \mathcal{N}^{*}\left(E, t_{0}\right)(t):=\bigcap_{\rho>0} \mathcal{N}\left(E_{\rho}^{+}, t_{0}\right)(t),
\end{aligned}
$$

where $E_{\rho}^{ \pm}=\left\{d_{E} \leqslant \pm \rho\right\}$

We recall the following result, proved in [6] (see also [22] for the case of the motion by mean curvature).

ThEOREM 2.4 Assume that $G(x, t)=\int_{0}^{t} g(x, s) \mathrm{d} s$ with $g$ continuous. Then $\mathcal{M}^{*}\left(E, t_{0}\right)(t)=$ $\mathcal{N}^{*}\left(E, t_{0}\right)(t)$ and $\mathcal{M}_{*}\left(E, t_{0}\right)(t)=\mathcal{N}_{*}\left(E, t_{0}\right)(t)$ for any $E \subset \mathbb{R}^{N}$ and $t \geqslant t_{0}$. Moreover, the set $\mathcal{M}^{*}\left(E, t_{0}\right)(t) \backslash \mathcal{M}_{*}\left(E, t_{0}\right)(t)$ coincides with the zero level-set of the viscosity solution of the parabolic equation corresponding to (1).

The parabolic equation mentioned here is equation (21) or (22). In the following, we shall omit the explicit dependence of barriers on $t_{0}$ whenever $t_{0}=0$. 


\subsection{Anisotropic total variation}

The total variation of a function $w \in L^{1}(\Omega)$ is defined as

$$
\sup \left\{\int_{\Omega} u(x) \operatorname{div} \psi(x) \mathrm{d} x: \psi \in C_{0}^{1}\left(\Omega ; \mathbb{R}^{N}\right),|\psi(x)| \leqslant 1 \forall x \in \Omega\right\} .
$$

It turns out that it is finite if and only if the distributional derivative $D w$ is a bounded Radon measure. In this case, the total variation is equal to the variation $|D w|(\Omega)=\int_{\Omega}|D w|$ of the measure $D w$, and $w$ belongs to the space $B V(\Omega)$ of functions with bounded variation.

Given a couple $\left(\phi, \phi^{\circ}\right)$ of mutually polar norms in $\mathbb{R}^{N}$ (an anisotropy), one defines in the same way the anisotropic total variation

$$
\int_{\Omega} \phi^{\circ}(D w)=\sup \left\{\int_{\Omega} u(x) \operatorname{div} \psi(x) \mathrm{d} x: \psi \in C_{0}^{1}\left(\Omega ; \mathbb{R}^{N}\right), \phi(\psi(x)) \leqslant 1 \forall x \in \Omega\right\} .
$$

Clearly, it is finite if and only if $w \in B V(\Omega)$. If $w=\chi_{E}$, the characteristic function of a measurable set $E$, then $w \in B V(\Omega)$ if and only if $E$ is a set of finite perimeter in $\Omega$ (a Caccioppoli set). In this case, one can define a reduced boundary $\partial^{*} E$ (which is $\mathcal{H}^{N-1}$-equivalent to the measure-theoretical boundary, that is, the set of points where $E$ has Lebesgue density neither 0 nor 1 ), on which a normal unit vector $v_{E}(x)$ is well defined, and $D \chi_{E}=v_{E} \mathcal{H}^{N-1}\left\llcorner\partial^{*} E\right.$. Then

$$
\int_{\Omega}\left|D \chi_{E}\right|=\mathcal{H}^{N-1}\left(\partial^{*} E\right) \quad \text { and } \quad \int_{\Omega} \phi^{\circ}\left(D \chi_{E}\right)=\int_{\partial^{*} E} \phi^{\circ}\left(v_{E}(x)\right) \mathrm{d} \mathcal{H}^{N-1}(x) .
$$

See [20, 3] for more details.

\section{The implicit time discretization}

Let $\Omega$ be a bounded, convex, open subset of $\mathbb{R}^{N}$. Let $G \in C^{0}\left([0,+\infty) ; L^{\infty}(\Omega)\right)$ and let $\omega_{G, T}$ be its modulus of continuity in $[0, T]$. Let $\left(\phi, \phi^{\circ}\right)$ be the anisotropy, which we assume to be smooth and elliptic. Let $E \subseteq \mathbb{R}^{N}$. Given $s>t \geqslant 0$, let $w$ denote the unique solution of

$$
\min _{w \in L^{2}(\Omega)} \int_{\Omega} \phi^{\circ}(D w)+\frac{1}{2(s-t)} \int_{\Omega}\left(w(x)-d_{E}(x)-G(x, s)+G(x, t)\right)^{2} \mathrm{~d} x .
$$

We let $T_{t, s}(E)=\{x \in \Omega: w(x)<0\}$. The existence and uniqueness of $w$ minimizing (3) does not raise any difficulty, since the energy which is minimized is trivially strictly convex, and lower-semicontinuous in $L^{2}(\Omega)$.

Notice that the set $T_{t, s}(E)$ is the minimizer of a prescribed curvature problem, with bounded mean curvature. Indeed, reasoning as in [12, 11, 4], one can check that this set is a solution of the variational problem

$$
\min \left(\int_{\Omega \cap \partial^{*} F} \phi^{\circ}\left(v_{F}(x)\right) \mathrm{d} \mathcal{H}^{N-1}(x)+\frac{1}{s-t} \int_{F}\left(d_{E}(x)+G(x, s)-G(x, t)\right) \mathrm{d} x\right),
$$

where the minimum is taken over the subsets $F$ of $\Omega$ of finite perimeter. It follows that the set $T_{t, s}(E)$ has boundary of class $C^{1, \alpha}$ inside $\Omega$, outside a compact singular set of zero $\mathcal{H}^{N-1}$ dimension [1] (when $N=2$, the set $T_{t, s}(E)$ has boundary of class $C^{1,1}$ ). The variational problem 
above is a generalization of the approach proposed in [1, 25], for building mean curvature flows without driving terms, through an implicit time discretization.

For $s=t+h$, the Euler-Lagrange equation for $w$ at a point $x \in \partial T_{t, t+h}(E)$ formally reads

$$
d_{E}(x)=-h\left(\kappa_{\phi}(x)+\frac{G(x, t+h)-G(x, t)}{h}\right),
$$

with $\kappa_{\phi}$ being the $\phi$-curvature of $\partial T_{t, t+h}(E)$ at $x$, so that it corresponds to an implicit time discretization of (1). Observe also that this approximation is monotone: indeed, if $E \subseteq E^{\prime}$ then $d_{E} \geqslant d_{E^{\prime}}$, which yields $w \geqslant w^{\prime}$, where $w$ and $w^{\prime}$ are the solutions of (3) for the distance functions $d_{E}$ and $d_{E^{\prime}}$ respectively. We deduce that $\{w<0\} \subseteq\left\{w^{\prime}<0\right\}$, that is, $T_{t, s}(E) \subseteq T_{t, s}\left(E^{\prime}\right)$.

We will soon show (Thm. 3.3 that this scheme is also consistent, in some sense, with the evolution (1). Before this, let us prove that it is independent of $\Omega$, in the sense that if $\partial E \subset \Omega$, then for $s-t$ small enough the set $T_{t, s}(E)$ is also the zero sublevel-set of any function $w^{\prime}$ solving (3) in any larger open set $\Omega^{\prime} \supseteq \Omega$. This justifies ignoring the dependence on $\Omega$ in our notation. Here and in the rest of the paper we shall assume that $G$ is defined in the whole space: $G \in C^{0}\left([0,+\infty) ; L^{\infty}\left(\mathbb{R}^{N}\right)\right)$.

PRoposition 3.1 For any $\delta, T>0$, there exists $h_{0}>0$ such that if $E$ is a closed set with compact boundary $\partial E \subset \Omega$ such that $\operatorname{dist}_{\phi}(\partial \Omega, \partial E) \geqslant \delta$, then for any $h \leqslant h_{0}$ and $t \leqslant T$, the set $T_{t, t+h}(E)$ is the same whether computed in $\Omega$ or in any larger open set $\Omega^{\prime} \supseteq \Omega$.

Before proving this proposition, we show a result that allows us to control in some uniform way the speed at which an initial Wulff shape $\left\{\phi\left(x-x_{0}\right) \leqslant \rho\right\}$ decreases in an iteration of the algorithm. The convexity of $\Omega$ is needed in the proof of this result.

LEMMA 3.2 Let $x_{0} \in \Omega$ and $\rho>0$, and let $t \geqslant 0$. Let $w$ solve

$$
\min _{w \in L^{2}(\Omega)} \int_{\Omega} \phi^{\circ}(D w)+\frac{1}{2 h} \int_{\Omega}\left(w(x)-\left(\phi\left(x-x_{0}\right)-\rho\right)-G(x, t+h)+G(x, t)\right)^{2} \mathrm{~d} x .
$$

Then

$$
w(x) \leqslant \begin{cases}\phi\left(x-x_{0}\right)+h \frac{N-1}{\phi\left(x-x_{0}\right)}+\Delta_{h}(t)-\rho & \text { if } \phi\left(x-x_{0}\right) \geqslant \sqrt{h(N+1)}, \\ \sqrt{h} \frac{2 N}{\sqrt{N+1}}+\Delta_{h}(t)-\rho & \text { otherwise, }\end{cases}
$$

where $\Delta_{h}(t):=\|G(\cdot, t+h)-G(\cdot, t)\|_{L^{\infty}(\Omega)}$.

Proof. The Euler-Lagrange equation for (4) can be written as follows: there exists a field $z \in$ $L^{\infty}\left(\Omega ; \mathbb{R}^{N}\right)$, with $z \in \partial \phi^{\circ}(\nabla w)$ a.e. and $z \cdot v_{\Omega}=0$ on $\partial \Omega$, such that

$$
w(x)-\phi\left(x-x_{0}\right)+\rho-G(x, t+h)+G(x, t)-h \operatorname{div} z(x)=0
$$

(see for instance [11, 4]).

Let $\bar{w}$ denote the function on the right-hand side of $(5)$. Let $\bar{z}$ be the field given by

$$
\bar{z}(x)= \begin{cases}\frac{x-x_{0}}{\phi\left(x-x_{0}\right)} & \text { if } \phi\left(x-x_{0}\right) \geqslant \sqrt{h(N+1)}, \\ \left(1-\left(\frac{\phi\left(x-x_{0}\right)}{\sqrt{h(N+1)}}-1\right)^{2}\right) \frac{x-x_{0}}{\phi\left(x-x_{0}\right)} & \text { otherwise. }\end{cases}
$$


One checks, as in [11, App. B], that $\bar{z} \in \partial \phi^{\circ}(\nabla \bar{w}(x))$ a.e., and

$$
\frac{\bar{w}(x)-\phi\left(x-x_{0}\right)+\rho}{h}-\operatorname{div} \bar{z}(x)=\frac{\Delta_{h}(t)}{h}
$$

a.e. in $\Omega$. Moreover, if $x \in \partial \Omega$, then $\bar{z}(x) \cdot v_{\Omega}(x)$ has the sign of $\left(x-x_{0}\right) \cdot v_{\Omega}(x)$, which is nonnegative since $\Omega$ is convex. By definition of $\Delta_{h}(t)$, we deduce that $\bar{w}$ is a supersolution for (6). It follows that $\bar{w} \geqslant w$ a.e. in $\Omega$ : indeed, we have

$$
\begin{aligned}
\int_{\Omega}\left[(w-\bar{w})^{+}\right]^{2} & =\int_{\{w>\bar{w}\}}(w-\bar{w})(\operatorname{div} z-\operatorname{div} \bar{z}) \\
& =\int_{\partial \Omega \cap\{w>\bar{w}\}}(w-\bar{w})(z-\bar{z}) \cdot v_{\Omega}-\int_{\{w>\bar{w}\}}(\nabla w-\nabla \bar{w}) \cdot(z-\bar{z}) \\
& \leqslant-\int_{\partial \Omega \cap\{w>\bar{w}\}}(w-\bar{w}) \bar{z} \cdot v_{\Omega} \leqslant 0,
\end{aligned}
$$

which shows the desired inequality.

Proof of Proposition 3.1. We assume $E \subset \Omega$, the proof in the case $\mathbb{R}^{N} \backslash E \subset \Omega$ being identical. Let $w$ solve

$$
\min _{w \in L^{2}(\Omega)} \int_{\Omega} \phi^{\circ}(D w)+\frac{1}{2 h} \int_{\Omega}\left(w(x)-d_{E}(x)-G(x, t+h)+G(x, t)\right)^{2} \mathrm{~d} x,
$$

and let $x \in \Omega$ with $d_{E}(x) \geqslant \delta / 2$. One has $d_{E} \geqslant \delta / 2-\phi(\cdot-x)$ in $\Omega$. Invoking Lemma 3.2, we deduce that $w(x) \geqslant \delta / 2-\Delta_{h}(t)-2 N \sqrt{h} / \sqrt{N+1}$. Hence if $h_{0} \leqslant 1$ is such that $\omega_{G, T+1}\left(h_{0}\right)+$ $2 N \sqrt{h_{0}} / \sqrt{N+1} \leqslant \delta / 4$, we find that when $h \leqslant h_{0}$ we have $w(x) \geqslant \delta / 4$.

Let now $\Omega^{\prime} \supseteq \Omega$. If $h \leqslant h_{0}$, we have in particular $d_{E}(x)+G(x, t+h)-G(x, t) \geqslant \delta / 4$ for any $x \in \Omega^{\prime} \backslash \Omega, t \leqslant T$. We can hence reproduce the proof of Corollary A.2 in [12], which shows that if $w^{\prime}$ is the solution of the same problem as $w$, but in $\Omega^{\prime}$ instead of $\Omega$, then $w^{\prime} \wedge(\delta / 4)$ is equal to $w \wedge(\delta / 4)$ in $\Omega$ and to $\delta / 4$ in $\Omega^{\prime} \backslash \Omega$. Thus $\{w<0\}=\left\{w^{\prime}<0\right\}$. Observe that in this proof, the larger domain $\Omega^{\prime}$ does not need to be convex.

The previous proposition allows us to define, in a unique and intrinsic way, the evolution $T_{t, t+h}(E)$ in $\mathbb{R}^{N}$, for any $t \geqslant 0$ and $h>0$, of a set $E$ with compact boundary $\partial E \Subset \mathbb{R}^{N}$, by considering the corresponding set computed in a ball with radius large enough. Therefore, from now on we shall assume $\partial E \Subset \mathbb{R}^{N}$ and we shall omit the dependence on $\Omega$ in the construction of the limit flow. We now prove our main consistency result.

THEOREM 3.3 Let $E(t), t \in\left[t_{0}, t_{1}\right]$, be a superflow of (1). Then there exists $h_{0}$ such that $T_{t, t+h}(E(t)) \supseteq E(t+h)$ for any $h<h_{0}$ and $t$ with $t_{0} \leqslant t<t+h \leqslant t_{1}$. Moreover, if $E(t)$ is a subflow of (1), then $T_{t, t+h}(E(t)) \subseteq E(t+h)$ for $h$ small enough.

Proof. Let $A \subset \mathbb{R}^{N}$ be the open set associated to the superflow $E(t)$ (cf. Definition 2.1) and let $\Omega$ be a bounded, convex open set with $A \Subset \Omega$.

We first observe that there exists $\varepsilon>0$ such that $C:=\left\{(x, t): t_{0} \leqslant t \leqslant t_{1},|d(x, t)| \leqslant \varepsilon\right\} \subset$ $A \times\left[t_{0}, t_{1}\right]$. Since $d(\cdot, t)$ is uniformly bounded in $C^{2}(A)$, we can also assume, possibly reducing $\varepsilon$, that $E(t)$ satisfies for all $t$ an interior and exterior $\varepsilon W_{\phi}$-condition. Given $t, h$ with $t_{0} \leqslant t<t+h$ $\leqslant t_{1}$, we build from $d(\cdot, t+h)$ a supersolution for problem (3). Consider a smooth increasing 
function $\psi: \mathbb{R} \rightarrow \mathbb{R}$ with $\psi(s) \geqslant s$ and $\psi(s)=s$ for $|s| \leqslant \varepsilon / 2$. We set $v(x):=\psi(d(x, t+h))$ for $x \in B=\{|d(\cdot, t)|<\varepsilon\}$. Then, for $x \in B$, from (2) it follows

$$
\begin{aligned}
\frac{v(x)-d_{E(t)}(x)-G(x, t+h)+G(x, t)}{h} & \geqslant \frac{d(x, t+h)-d(x, t)-G(x, t+h)+G(x, t)}{h} \\
& \geqslant \frac{1}{h} \int_{t}^{t+h} \operatorname{div} \nabla \phi^{\circ}(\nabla d)(x, s) \mathrm{d} s+\delta .
\end{aligned}
$$

Let now $\omega$ be a modulus of continuity for $\operatorname{div} \nabla \phi^{\circ}(\nabla d)$ in $C$. We find

$$
\frac{v(x)-d_{E(t)}(x)-G(x, t+h)+G(x, t)}{h} \geqslant \operatorname{div} \nabla \phi^{\circ}(\nabla d)(x, t+h)+\delta-\omega(h) .
$$

Observe that for any $x \in B$ we have $\nabla v(x)=\psi^{\prime}(d(x, t+h)) \nabla d(x, t+h)$, so that (recall that $\nabla \phi^{\circ}$ is 0-homogeneous) $\nabla \phi^{\circ}(\nabla v(x))=\nabla \phi^{\circ}(\nabla d(x, t+h))$, hence $\operatorname{div} \nabla \phi^{\circ}(\nabla d)(x, t+h)=$ $\operatorname{div} \nabla \phi^{\circ}(\nabla v)(x)$. Therefore, if $h$ is small enough so that $\omega(h) \leqslant \delta$, we get

$$
\frac{v(x)-d_{E(t)}(x)-G(x, t+h)+G(x, t)}{h} \geqslant \operatorname{div} \nabla \phi^{\circ}(\nabla v)(x) .
$$

Let $w$ solve (3), with $E=E(t)$ and $s=t+h$. We will show that we may choose $\psi$ in order to have $v \geqslant w$ on $\partial B$, so that $v$ is a supersolution for the problem

$$
\min \left\{\int_{B} \phi^{\circ}(D u)+\frac{1}{2 h} \int_{B}\left(u(x)-d_{E(t)}(x)-G(x, t+h)+G(x, t)\right)^{2} \mathrm{~d} x: u=w \text { on } \partial B\right\}
$$

(which is solved by $w$ ). We will deduce that $v \geqslant w$ in $B$, hence $\{w<0\} \supseteq\{v<0\}=$ $\{d(\cdot, t+h)<0\}$, that is, $T_{t, t+h}(E(t)) \supseteq E(t+h)$.

First of all, $d$ is uniformly continuous in time, so that if $h$ is small enough, one has $d(x, t+h) \geqslant$ $3 \varepsilon / 4$ if $d(x, t)=\varepsilon$. If $M>\operatorname{diam} \Omega$, then $M \geqslant w$ in $\Omega$. We may choose a function $\psi$ with $\psi(3 \varepsilon / 4) \geqslant M$, so that $v(x) \geqslant M \geqslant w(x)$ if $d(x, t)=\varepsilon$.

On the other hand, since $E(t)$ satisfies the interior $\varepsilon W_{\phi}$-condition, one deduces from Lemma 3.2 that $w(x) \leqslant 2 N \sqrt{h} / \sqrt{N+1}+\Delta_{h}(t)-\varepsilon$ whenever $d(x, t)=-\varepsilon$. We observe that $\Delta_{h}(t) \rightarrow 0$ as $h \rightarrow 0$ uniformly in $\left[t_{0}, t_{1}\right]$. Hence if $h$ is small enough, we find that $w(x) \leqslant-3 \varepsilon / 4$. We can choose $\psi$ such that $\psi(s) \geqslant-3 \varepsilon / 4$ for any $s$, so that $v(x) \geqslant w(x)$ if $d(x, t)=-\varepsilon$. We conclude that $v \geqslant w$ on $\partial B$. Hence $v$ is a supersolution for (3), which implies $T_{t, t+h}(E(t)) \supseteq E(t+h)$.

If $E(t)$ is a subflow, we can reproduce the same proof to show that $T_{t, t+h}(E(t)) \subseteq E(t+h)$.

We deduce the following comparison result for sub/superflows.

COROLlaRY 3.4 Assume that $E_{1}(t), E_{2}(t)$ are respectively a superflow and subflow of (1) on $\left[t_{0}, t_{1}\right]$ such that $E_{1}\left(t_{0}\right) \subseteq E_{2}\left(t_{0}\right)$. Then $E_{1}(t) \subseteq E_{2}(t)$ for all $t \in\left[t_{0}, t_{1}\right]$.

Proof. By the previous theorem, there exists $h_{0}$ such that $T_{t, t+h}\left(E_{1}(t)\right) \supseteq E_{1}(t+h)$ and $T_{t, t+h}\left(E_{2}(t)\right) \subseteq E_{2}(t+h)$ for any $t \in\left[t_{0}, t_{1}-h\right]$, as soon as $h \leqslant h_{0}$. Hence, if $t \in\left[t_{0}, t_{1}\right]$, we just let $n \geqslant 1$ be such that $\left(t-t_{0}\right) / n=h \leqslant h_{0}$. Then, letting $t_{k}=t_{0}+k h$, one can easily check by induction that $E_{1}\left(t_{k}\right) \subseteq T_{t_{k-1}, t_{k}}\left(E_{1}\left(t_{k-1}\right)\right) \subseteq T_{t_{k-1}, t_{k}}\left(E_{2}\left(t_{k-1}\right)\right) \subseteq E_{2}\left(t_{k}\right)$ for any $1 \leqslant k \leqslant n$, which implies the assertion since $t=t_{n}$. 
REMARK 3.5 It could be interesting, from a numerical analysis point of view, to modify slightly the algorithm presented in this paper by introducing a threshold $S>0$ and replace in problem (3) the distance function $d_{E}$ with a truncated distance function $\left(-S \wedge d^{E}\right) \vee S$. Almost all of the results presented in this paper would remain identical (in particular, the consistency still holds). Only the comparison results in Section 4.2 are not completely clear, since they rely on comparisons of the true distance functions. However, it is reasonable to believe that in the limit $h \rightarrow 0$ also these results hold.

\section{Convergence of the algorithm}

In this section, we study the limit of the iterates of our variational algorithm as the time-step goes to zero. First (Section 4.1), under some local boudedness assumption on the forcing term, we find a limit which has some continuity properties (Props. 4.3 and 4.4), and we show that it is consistent with other notions of weak solutions (Cor. 4.6, and with further assumptions on the forcing term, Props. 4.8 and 4.9 .

Then in 4.2 assuming some spatial regularity $(18)$ of the forcing term, we find a comparison principle for our limits (however, with some limitation), which allows us to build level-set solutions starting from an arbitrary bounded uniformly continuous function (Section 4.3).

\subsection{The discrete flow and its limit}

Given $E \subset \mathbb{R}^{N}$, closed with compact boundary, and $h>0$, we define the "tube" $E_{h} \subset \mathbb{R}^{N} \times$ $[0,+\infty)$ as follows:

$$
E_{h}(t):=T_{[t / h] h-h,[t / h] h} \cdots T_{2 h, 3 h} T_{h, 2 h} T_{0, h}(E),
$$

where $[x]$ denotes the integer part of $x$. We then define $E_{h}:=\bigcup_{t \geqslant 0} E_{h}(t) \times\{t\}$.

There exists a sequence $\left(h_{n}\right)_{n} \geqslant 1$ such that both $E_{h_{n}}$ and $\mathbb{R}^{N} \times[0,+\infty) \backslash E_{h_{n}}={ }^{c} E_{h_{n}}$ converge in the Hausdorff distance (locally in time) to $E^{*}$ and ${ }^{c} E_{*}$ respectively. Such convergence is equivalent to the locally uniform convergence, in $\mathbb{R}^{N} \times[0,+\infty)$, of the distance functions $\operatorname{dist}\left((x, t), E_{h_{n}}\right)$ and $\operatorname{dist}\left((x, t),{ }^{c} E_{h_{n}}\right)$ to the $\operatorname{distance}$ functions $\operatorname{dist}\left((x, t), E^{*}\right)$ and $\operatorname{dist}\left((x, t),{ }^{c} E_{*}\right)$ (see [11, App. A]). In particular, for any $(x, t) \in E^{*}$ (resp., $\left.{ }^{c} E_{*}\right)$, there exist $\left(x_{n}, t_{n}\right) \in E_{h_{n}}$ (resp., ${ }^{c} E_{h_{n}}$ ) such that $\left(x_{n}, t_{n}\right) \rightarrow(x, t)$, and if $\left(x_{n}, t_{n}\right) \in E_{h_{n}}$ (resp., $\left.{ }^{c} E_{h_{n}}\right)$ and converge to some point $(x, t) \in \Omega \times$ $[0,+\infty)$, then $(x, t) \in E^{*}$ (resp., $\left.{ }^{c} E_{*}\right)$. Below, we denote by $(h)_{h>0}$ the sequence $\left(h_{n}\right)_{n \geqslant 1}$.

Clearly, $E_{*}$ is open while $E^{*}$ is closed, and $E_{*} \subset E^{*}$. For any $t \geqslant 0$, we denote by $E^{*}(t)$ (resp. $\left.E_{*}(t)\right)$ the section $\left\{x:(x, t) \in E^{*}\right\}$ (resp. $\left\{x:(x, t) \in E_{*}\right\}$ ). For any $t \geqslant 0$, we let $\Gamma(t)=E^{*}(t) \backslash E_{*}(t)$, which in some sense is our generalized evolution starting from $\partial E$.

From the definition of $E_{*}, E^{*}$ it follows that

$$
E_{*}(0) \subseteq \operatorname{int}(E), \quad E \subseteq E^{*}(0),
$$

in particular $\Gamma(0) \supseteq \partial E$. If $F(t)$ is a superflow on $\left[t_{0}, t_{1}\right]$ such that $F\left(t_{0}\right) \subset E_{*}\left(t_{0}\right)$, since $\operatorname{dist}\left(F\left(t_{0}\right) \times\left\{t_{0}\right\},{ }^{c} E_{*}\right)>0$ (as $F(t)$ is assumed to be closed for any time $t$, and $E_{*}$ is open), one sees that for $h$ small enough, $F\left(t_{0}\right) \subset E_{h}\left(\left[t_{0} / h\right] h\right) \cap E_{h}\left(\left[t_{0} / h\right] h+h\right)$. It then follows from Theorem 3.3 that (if $h$ is enough small) $F(t) \subset E_{h}(t)$ for any $t \in\left[t_{0}, t_{1}\right]$, and passing to the limit we get $F \subset E_{*} \cap\left(\mathbb{R}^{N} \times\left[t_{0}, t_{1}\right]\right)$. Hence $E_{*}$ satisfies a comparison principle for superflows that start inside, and analogously $E^{*}$ satisfies a comparison principle for subflows starting outside, so that we have shown the following: 
Proposition 4.1 The set-valued functions $E_{*}(\cdot)$ and $E_{*}(\cdot)$ are barriers on $[0,+\infty)$ with respect to $\mathcal{F}^{+}$and $\mathcal{F}^{-}$respectively, that is, $E_{*} \in \mathcal{B}^{+}$and $E^{*} \in \mathcal{B}^{-}$. In particular, from Definition 2.3 that for all $t \geqslant 0$,

$$
E_{*}(t) \supseteq \mathcal{M}_{*}\left(E_{*}(0)\right)(t) \text { and } \quad E^{*}(t) \subseteq \mathcal{M}^{*}\left(E^{*}(0)\right)(t) .
$$

On the other hand, it is not clear if we also have $E_{*} \in \mathcal{B}^{-}$and $E^{*} \in \mathcal{B}^{+}$.

Let us now show that $E_{*}(0)=\operatorname{int}(E)$ and $E^{*}(0)=E$, so that we can substitute $E_{*}(0)$ and $E^{*}(0)$ with $E$ in $(9)$. In order to do so, we further require that the function $G$ satisfies the following regularity assumption: for any $T>0$ there exists a constant $C(T)$ such that

$$
\left|\frac{G(x, s)-G(y, s)-G(x, t)+G(y, t)}{s-t}\right| \leqslant C(T)
$$

for any $s, t \leqslant T$. Note that this is equivalent to requiring that $G$ can be written as $G(x, t)=$ $G_{1}(t)+G_{2}(x, t)$ with $G_{1} \in C^{0}([0,+\infty))$ and $G_{2} \in \operatorname{Lip}_{\text {loc }}\left([0,+\infty) ; L^{\infty}\left(\mathbb{R}^{N}\right)\right)$.

We first construct explicit super/subflows starting from a Wulff shape $W_{\phi}\left(x_{0}, r\right)$ of radius $r>0$ (or its complement), at time $t \geqslant 0$. More precisely, we construct superflows $W_{x_{0}, t, r}^{+}(s)$, with $s \in$ $[t,+\infty)$, starting from $W_{\phi}\left(x_{0}, r\right)$ at time $t$, which are smooth on $[t, t+\tau]$ and vanish after time $t+\tau$, where the duration $\tau$ depends only on $r$, and such that $T_{s, s+h}\left(W_{x_{0}, t, r}^{+}(s)\right) \supseteq W_{x_{0}, t, r}^{+}(s+h)$ for all $h>0$ and $s \geqslant t$.

LEMMA 4.2 Let $x_{0} \in \mathbb{R}^{N}$ and $r>0$. Define

$$
d_{ \pm}(x, s):= \pm\left(\phi\left(x-x_{0}\right)-r+\frac{s-t}{2 \tau} r\right)+G\left(x_{0}, s\right)-G\left(x_{0}, t\right)
$$

for $(x, s) \in \mathbb{R}^{n} \times[t, t+\tau]$, where $\tau$ is such that

$$
\begin{aligned}
& \omega_{G, t+\tau}(\tau) \leqslant \frac{r}{4}, \\
& \tau \leqslant \frac{r^{2}}{2(C(t+\tau)+4(N-1))} \wedge \frac{r^{2}}{16(N+1)},
\end{aligned}
$$

where $\omega_{G, t+\tau}$ is as before a modulus of continuity of $G$ on $[0, t+\tau]$, and $C(\cdot)$ is the constant appearing in [10). Let $W_{x_{0}, t, r}^{+}(s):=\left\{d_{+}(\cdot, s) \leqslant 0\right\}$ when $s \in[t, t+\tau]$, and $W_{x_{0}, t, r}^{+}(s):=\emptyset$ for $s>t+\tau$. Then $T_{s, s+h}\left(W_{x_{0}, t, r}^{+}(s)\right) \supseteq W_{x_{0}, t, r}^{+}(s+h)$ for any $s \geqslant t$ and $h>0$. On the other hand, if $W_{x_{0}, t, r}^{-}(s):=\left\{d_{-}(\cdot, s) \leqslant 0\right\}$ when $s \in[t, t+\tau]$, and $W_{x_{0}, t, r}^{-}(s):=\mathbb{R}^{N}$ for $s>t+\tau$, then $T_{s, s+h}\left(W_{x_{0}, t, r}^{-}(s)\right) \subseteq W_{x_{0}, t, r}^{-}(s+h)$ for any $s \geqslant t$ and $h>0$.

Notice that, letting $\tau(r)$ be the maximal time $\tau$ satisfying $(12)$ and $(13)$ for a given $r>0$, we have $\tau(r)>0$ and

$$
\lim _{r \rightarrow \infty} \tau(r)=+\infty .
$$

Notice also that the condition $\omega_{G, t+\tau}(\tau) \leqslant r / 4$ ensures the inclusion $W_{\phi}\left(x_{0}, r / 4\right) \subseteq W_{x_{0}, t, r}^{+}(s)$ for $s \in[t, t+\tau]$, hence in particular the set $W_{x_{0}, t, r}^{+}(s)$ is nonempty. In fact, one could check that $W_{x_{0}, t, r}^{+}$is a superflow (in the sense of Definition 2.1) on $[t, t+\tau]$, while $W_{x_{0}, t, r}^{-}$is a subflow. Thus, the conclusion would follow from Theorem 3.3 , at least for $h$ small enough. The statement of Lemma 4.2 is slightly more precise, as it holds without any restriction on $h$ so that the superflow property which is shown is, in particular, uniform in $x_{0}$. 
Proof. Let $s \in[t, t+\tau]$ and $h>0$. If $s+h>t+\tau$, then $W_{x_{0}, t, r}^{+}(s)=\emptyset$ so that the statement is obvious, hence we may assume $s+h \leqslant t+\tau$. For any $x \in \mathbb{R}^{N}$, by 10$]$ we have

$$
\begin{aligned}
d_{+}(x, s) & +G(x, s+h)-G(x, s) \\
& =\phi\left(x-x_{0}\right)-r+G\left(x_{0}, s\right)-G\left(x_{0}, t\right)+G(x, s+h)-G(x, s)+\frac{s-t}{2 \tau} r \\
& \leqslant \phi\left(x-x_{0}\right)-r+G\left(x_{0}, s+h\right)-G\left(x_{0}, t\right)+\frac{s-t}{2 \tau} r+C(t+\tau) h .
\end{aligned}
$$

Let now $\Omega$ be an open bounded subset of $\mathbb{R}^{N}$ which is big enough to guarantee that the set $T_{s, s+h}\left(W_{x_{0}, t, r}^{+}(s)\right)$ does not depend on $\Omega$ (Prop. 3.1). Hence the solution $w$ of (3), with $E$ replaced by $W_{x_{0}, t, r}^{+}(s)$ and $(t, s)$ replaced by $(s, s+h)$, is less than the solution of

$$
\begin{aligned}
\min _{v \in B V(\Omega)}( & \int_{\Omega} \phi^{\circ}(D v) \\
& \left.+\frac{1}{2 h} \int_{\Omega}\left(v-\phi\left(x-x_{0}\right)+r-G\left(x_{0}, s+h\right)+G\left(x_{0}, t\right)-\frac{s-t}{2 \tau} r-C(t+\tau) h\right)^{2} \mathrm{~d} x\right),
\end{aligned}
$$

which in turn (as shown in the proof of Lemma 3.2) is less than the function

$$
\left\{\begin{array}{r}
\phi\left(x-x_{0}\right)+h \frac{N-1}{\phi\left(x-x_{0}\right)}-r+G\left(x_{0}, s+h\right)-G\left(x_{0}, t\right)+\frac{s-t}{2 \tau} r+C(t+\tau) h \\
\quad \text { if } \phi\left(x-x_{0}\right) \geqslant \sqrt{h(N+1)} \\
\sqrt{h} \frac{2 N}{\sqrt{N+1}}-r+G\left(x_{0}, s+h\right)-G\left(x_{0}, t\right)+\frac{s-t}{2 \tau} r+C(t+\tau) h \quad \text { otherwise. }
\end{array}\right.
$$

Hence, we see that

$$
w(x) \leqslant d_{+}(x, s+h)+h \frac{N-1}{\phi\left(x-x_{0}\right)}-h \frac{r}{2 \tau}+C(t+\tau) h
$$

when $\phi\left(x-x_{0}\right) \geqslant \sqrt{h(N+1)}$. Now, since $\tau \leqslant r^{2} /(16(N+1))$ and $h \leqslant \tau$, we get $r / 4 \geqslant$ $\sqrt{h(N+1)}$ so that we can replace the last condition with the stronger condition $\phi\left(x-x_{0}\right) \geqslant r / 4$. On the other hand, if both $\phi\left(x-x_{0}\right) \geqslant r / 4$ and $\tau \leqslant r^{2} /(2(C(t+\tau)+4(N-1)))$, then

$$
\frac{r}{2 \tau} \geqslant \frac{C(t+\tau)+4(N-1)}{r} \geqslant C(t+\tau)+\frac{N-1}{\phi\left(x-x_{0}\right)},
$$

so that $w(x) \leqslant d_{+}(x, s+h)$. This shows that $T_{s, s+h}\left(W_{x_{0}, t, r}^{+}(s)\right) \supseteq W_{x_{0}, t, r}^{+}(s+h)$.

The proof of the similar assertion for $W_{x_{0}, t, r}^{-}$is analogous.

By the previous lemma, if $E_{h}(t) \supseteq W_{\phi}\left(x_{0}, r\right)$, then $E_{h}(t+n h) \supseteq W_{x_{0}, t, r}^{+}(t+n h)$ for any $n \geqslant 1$, and in particular $E_{h}(t+n h) \supseteq W_{\phi}\left(x_{0}, r / 4\right)$ as long as $n h \leqslant \tau(r)$. In other words, for any $r>0$, the inequality $n h \leqslant \tau(r)$ yields

$$
E_{h}(t+n h) \supseteq\left\{x: d_{E_{h}(t)}(x) \leqslant-r\right\} .
$$

This shows that the (discrete) evolution will not vanish "suddenly", in the sense that any subset in the interior of $E_{h}(t)$ remains in the interior of $E_{h}(s)$ for $s>t$ sufficiently close to $t$. 
We can easily deduce the same "semicontinuity" property for $E_{*}$ : indeed, if $d_{E_{*}(t)}(x)=-r$, then, for any $r^{\prime}<r, W_{\phi}\left(x, r^{\prime}\right) \subset E_{h}(t)$ as soon as $h$ is small enough, so that $W_{\phi}\left(x, r^{\prime} / 4\right) \subset$ $E_{h}(t+[\tau / h] h)$ for all $\tau<\tau\left(r^{\prime}\right)$. Letting first $h \rightarrow 0$ and then $r^{\prime} \rightarrow r$, we find that if $\tau<\tau(r)$, then

$$
E_{*}(t+\tau) \supseteq\left\{x: d_{E_{*}(t)}(x) \leqslant-r\right\} .
$$

In the same way we obtain

$$
E^{*}(t+\tau) \subseteq\left\{x: d_{E^{*}(t)}(x)<r\right\} .
$$

Moreover, one can easily verify that the same properties hold at $t=0$ with $E^{*}(t)$ replaced with $E$ and $E_{*}(t)$ replaced with $\operatorname{int}(E)$, where $E$ is the initial set. From (14) and (15) we also get

$$
\Gamma(t+\tau) \subseteq\left\{\operatorname{dist}_{\phi}(\cdot, \Gamma(t))<r\right\} .
$$

As a consequence, we obtain the following semicontinuity property for the tubes $E_{*}, E^{*}$.

Proposition 4.3 Assume that $G$ satisfies $[10)$. Let $E$ be a closed subset of $\mathbb{R}^{N}$ with compact boundary. Let $O, F$ be an open and a closed subset of $\mathbb{R}^{N}$ respectively. Let $t \geqslant 0$ and let $\left(\tau_{n}\right)_{n \geqslant 0}$ be a sequence of nonnegative numbers going to 0 . Then

- If ${ }^{c} E_{*}\left(t+\tau_{n}\right) \rightarrow{ }^{c} O$ in the Hausdorff sense, then $E_{*}(t) \subseteq O$,

- If $E^{*}\left(t+\tau_{n}\right) \rightarrow F$ in the Hausdorff sense, then $F \subseteq E^{*}(t)$,

In particular, any Hausdorff limit of a sequence $\Gamma\left(t+\tau_{n}\right)$ is contained in $\Gamma(t)$. Moreover, if $t=0$, we can replace $E_{*}(t)$ with $\operatorname{int}(E)$ in the first statement and $E^{*}(t)$ with $E$ in the second. In particular, choosing $\tau_{n} \equiv 0$, we get

$$
\operatorname{int}(E) \subseteq E_{*}(0) \subseteq E^{*}(0) \subseteq E,
$$

which implies, recalling [8], that

$$
E_{*}(0)=\operatorname{int}(E) \quad \text { and } \quad E^{*}(0)=E .
$$

Notice that $(17)$ shows that $\Gamma(0)=\partial E$.

Since $O \subset F$ in the above proposition, we also see that if $E^{*}(t)=\overline{E_{*}(t)}$, then $E^{*}(t+\tau) \rightarrow$ $E^{*}(t)$ in the Hausdorff sense as $\tau \rightarrow 0$, whereas if $E_{*}(t)=\operatorname{int}\left(E^{*}(t)\right)$, then ${ }^{c} E_{*}(t+\tau) \rightarrow{ }^{c} E_{*}(t)$, and if both are true, then $\Gamma(t+\tau)=E^{*}(t+\tau) \backslash E_{*}(t+\tau) \rightarrow \partial E^{*}(t)$ as $\tau \rightarrow 0$. To show this, one just needs to show that for any $x \in \partial E^{*}(t)$, there exist $x_{\tau} \in \Gamma(t+\tau)$ that converge to $x$ as $\tau \rightarrow 0$. We know that there exist $y_{\tau} \in E^{*}(t+\tau)$ and $z_{\tau} \notin E_{*}(t+\tau)$ such that both $y_{\tau}$ and $z_{\tau}$ converge to $x$. Then the segment $\left[y_{\tau}, z_{\tau}\right]$ must intersect $\Gamma(t+\tau)$ and any point $x_{\tau}$ in this intersection will have the desired property.

Notice also that if $E=\overline{\operatorname{int}(E)}$, then $\Gamma(t)=E^{*}(t) \backslash E_{*}(t)$ converges to $\partial E$ as $t \rightarrow 0$, in the Hausdorff sense.

The left continuity of the tubes $E_{*}, E^{*}$ is given by the following proposition.

Proposition 4.4 Assume that $G$ satisfies $(10)$. Let $E$ be a closed subset of $\mathbb{R}^{N}$ with compact boundary, and let $t>0$. Then ${ }^{c} E_{*}(t-\tau) \rightarrow{ }^{c} E_{*}(t)$ in the Hausdorff sense as $\tau \rightarrow 0$ with $\tau \geqslant 0$, while $E^{*}(t-\tau) \rightarrow E^{*}(t)$. Moreover, $\Gamma(t-\tau) \rightarrow \Gamma(t)$.

Proof (sketch). As for the previous proposition, one will deduce from (14) that if ${ }^{c} E_{*}\left(t-\tau_{n}\right) \rightarrow$ ${ }^{c} O$ in the Hausdorff sense, along a subsequence $\tau_{n}$ going to 0 , then $O \subseteq E_{*}(t)$. On the other hand, since ${ }^{c} E_{*}$ is closed in $\Omega \times[0,+\infty)$, one must have ${ }^{c} O \subseteq{ }^{c} E_{*}(t)$. Thus $O=E_{*}(t)$ and the 
conclusion follows. In the same way, (15) implies that if $E^{*}\left(t-\tau_{n}\right) \rightarrow F$ in the Hausdorff sense, then $E^{*}(t) \subseteq F$. From the closedness of $F^{*}$ we conclude in the same way that $F=E^{*}(t)$.

The last assertion follows from (16): first, any Hausdorff limit $F$ of a subsequence $\Gamma\left(t-\tau_{n}\right)=$ $E^{*}\left(t-\tau_{n}\right) \backslash E_{*}\left(t-\tau_{n}\right)$ is inside $\Gamma(t)=E^{*}(t) \backslash E_{*}(t)$, by the previous results. Now, since $\operatorname{dist}_{\phi}\left(\cdot, \Gamma\left(t-\tau_{n}\right)\right)$ converges uniformly to $\operatorname{dist}_{\phi}(\cdot, F)$ as $n \rightarrow \infty, \Gamma(t) \subseteq\left\{\operatorname{dist}_{\phi}(\cdot, F) \leqslant r\right\}$ for any $r>0$, hence it lies in $F$. Thus $F=\Gamma(t)$.

REMARK 4.5 Notice that in general we cannot expect the maps $t \mapsto E_{*}(t)$ and $t \mapsto E^{*}(t)$ to be continuous in the Hausdorff distance: indeed, this would prevent small disconnected parts from disappearing in finite time, a phenomenon which is known to happen even when $G \equiv 0$. On the other hand, these maps are likely to be continuous in the $L^{1}$-topology, under suitable assumptions on $G$ (when $G \equiv 0$ it is proved in [1, Thm. 4.4]).

From Propositions 4.1 and 4.3 , and in particular (9) and (17), we get the following corollary.

COROLlary 4.6 If $G$ satisfies condition (10), then

$$
\Gamma(t) \subseteq \mathcal{N}^{*}(E, 0)(t) \backslash \mathcal{M}_{*}(E, 0)(t) .
$$

In particular, as long as $\mathcal{N}^{*}(E, 0)(t) \backslash \mathcal{M}_{*}(E, 0)(t)$ has no interior (nonfattening condition), the motions $E^{*}(t)$ and $E_{*}(t)$ are uniquely defined and do not depend on the sequence along which the limits are obtained.

REMARK 4.7 Notice that, as long as the set $E$ has compact boundary, all the resuls of this section can be easily extended to functions $G$ which are only locally bounded in $x$, i.e. $G(x, t)=G_{1}(t)+$ $G_{2}(x, t)$ with $G_{1} \in C^{0}([0,+\infty))$ and $G_{2} \in \operatorname{Lip}_{\mathrm{loc}}\left([0,+\infty) ; L_{\mathrm{loc}}^{\infty}\left(\mathbb{R}^{N}\right)\right)$.

Proposition 4.8 If $G(x, t)=\int_{0}^{t} g(x, s) \mathrm{d} s$ with $g$ continuous, then $\Gamma(t)$ is contained in the zero level-set of the corresponding viscosity solution.

Proof. This follows immediately from Theorem 2.4 and Corollary 4.6 .

From Corollary 4.6 and [17, Section 3] we also have the following consistency result in the case of an $x$-independent forcing term.

Proposition 4.9 Let $G(x, t)=G(t) \in C^{0}([0,+\infty)$ ) and let $\phi(x)=|x|$ (i.e. isotropic mean curvature flow). Then $\Gamma(t)$ is contained in the minimal barrier solution defined in [17]. In particular, if $\partial E$ is of class $C^{2, \alpha}$, then $E^{*}(t)=\overline{E_{*}(t)}$ and $\Gamma(t)=\partial E^{*}(t)$ coincides with the unique (local in time) solution of (1) given in [17].

REMARK 4.10 As already pointed out in the introduction, viscosity theory can be applied under more general assumptions on $G$ than what is required in Proposition 4.8 (see [23, 24]). However, it is still not clear what is the relation between the limit set $\Gamma(t)$ and the zero level-set of such viscosity solutions, except for the particular case of an $x$-independent forcing term, where the equality holds for small times as a consequence of Proposition 4.9 (if $\partial E$ is regular enough).

\subsection{An inclusion principle}

Let us now consider the case where the driving term is the "time-derivative" of a function $G(x, t)$ that satisfies

$$
\left|\frac{G(x, s)-G(y, s)-G(x, t)+G(y, t)}{s-t}\right| \leqslant C(T)|x-y| .
$$


This condition is stronger than (10) (see also Remark 4.7) and is for instance true whenever $G(x, t)=G_{1}(t)+G_{2}(x, t)$ with $G_{1} \in C^{0}([0,+\infty))$ and $G_{2} \in C^{1}\left([0,+\infty) ; \operatorname{Lip}\left(\mathbb{R}^{N}\right)\right)$. In particular, all the results of Section 4.1 still hold under assumption (18).

Given a closed set $E \subset \mathbb{R}^{N}$ with nonempty compact boundary $\partial E$, we define the maximal existence time $T_{E}^{*} \in[0,+\infty]$ for the flow $E^{*}$ as the supremum of all times $t$ such that $E^{*}(t) \neq \varnothing$ and $E_{*}(t) \neq \mathbb{R}^{N}$. The fact that $T_{E}^{*}>0$ is ensured by Proposition 4.3 , whenever $\operatorname{int}(E) \neq \emptyset$.

Consider now two closed sets $E^{1}$ and $E^{2}$ with nonempty compact boundary, and assume $E^{1} \subset E^{2}$ and $D:=\operatorname{dist}_{\phi}\left(\partial E^{1}, \partial E^{2}\right)>0$. Notice that if $G$ depends only on time, then for each $z$ such that $\phi(z) \leqslant D$, we have $z+E^{1} \subset E^{2}$, so that $T_{0, h}\left(z+E^{1}\right) \subset T_{0, h}\left(E^{2}\right)$ for any $h>0$. Since $G$ does not depend on $x$, we get $T_{0, h}\left(z+E^{1}\right)=z+T_{0, h}\left(E^{1}\right)$. It follows that $W_{\phi}(0, D)+T_{0, h}\left(E^{1}\right) \subset T_{0, h}\left(E^{2}\right)$, which implies $\operatorname{dist}_{\phi}\left(\partial T_{0, h}\left(E^{1}\right), \partial T_{0, h}\left(E^{2}\right)\right) \geqslant D$. By induction, we deduce that $\operatorname{dist}_{\phi}\left(\partial E_{h}^{1}(t), \partial E_{h}^{2}(t)\right) \geqslant D$ for any $t \geqslant 0$ (where we set the distance equal to $+\infty$ if one of the two sets disappears).

For a general $G$ the estimate is slightly trickier, even if it follows the same idea. Assume $T^{*}=$ $\min \left\{T_{E^{1}}^{*}, T_{E^{2}}^{*}\right\}>0$ and let $T<T^{*}$. By Proposition 3.1. we can find a "large" bounded open set $\Omega \subset \mathbb{R}^{N}$ such that the sets $E_{h}^{1}(t)$ and $E_{h}^{2}(t)$ defined in (7) do not depend on $\Omega$ for $t \in[0, T]$ and $h$ small enough. In particular, we can assume that $\partial E_{h}^{1}(t)$ and $\partial E_{h}^{2}(t)$ remain at a positive distance from $\partial \Omega$ for any $t \in[0, T]$. Let $w_{1}, w_{2}$ be the solutions of the variational problem (3) for $t=0$ and $s=h$ with $d_{E}$ replaced by $d_{E^{1}}, d_{E^{2}}$ respectively. Notice that, for $z \in \mathbb{R}^{N}$, the set $z+T_{0, h}\left(E^{1}\right)=z+\left\{w_{1}<0\right\}$ coincides with $\left\{x \in z+\Omega: w_{1}(x-z)<0\right\}$, and the function $\tilde{w}_{1}(x)=w_{1}(x-z)$, defined in $z+\Omega$, is the solution of

$$
\min _{w \in L^{2}(z+\Omega)}\left(\int_{z+\Omega} \phi^{\circ}(D w)+\frac{1}{2 h} \int_{z+\Omega}\left(w(x)-d_{E^{1}}(x-z)-G(x-z, h)+G(x-z, 0)\right)^{2} \mathrm{~d} x\right) .
$$

Possibly enlarging $\Omega$, we can assume that both $w_{2}$ and $\tilde{w}_{1}$ are solutions of their respective variational problems in the same domain (for instance, $\Omega \cup(z+\Omega)$ ). Then, since $d_{E^{1}}(x-z)$ $\geqslant d_{E^{1}}(x)-\phi(z) \geqslant d_{E^{2}}(x)+D-\phi(z)$ and, by $(18),-G(x-z, h)+G(x-z, 0) \geqslant$ $-G(x, h)+G(x, 0)-C(T)|z| h$, one finds that $\tilde{w}_{1} \geqslant w_{2}+D-\phi(z)-C(T)|z| h$. In particular, if $\phi(z) \leqslant D /\left(1+h C^{\prime}(T)\right)$ with $C^{\prime}(T)=C(T) \sup _{z \neq 0}|z| / \phi(z)$, we get $\left\{\tilde{w}_{1} \leqslant 0\right\} \subset\left\{w_{2} \leqslant 0\right\}$, which in turn implies $\operatorname{dist}_{\phi}\left(\partial T_{0, h}\left(E^{1}\right), \partial T_{0, h}\left(E^{2}\right)\right) \geqslant D /\left(1+h C^{\prime}(T)\right)$. By an induction argument, we deduce that $\operatorname{dist}_{\phi}\left(\partial E_{h}^{1}(t), \partial E_{h}^{2}(t)\right) \geqslant D\left(1+h C^{\prime}(T)\right)^{-[t / h]}$ for any $t \in[0, T-h]$ and $h>0$ small enough. Observe that, as $h \rightarrow 0$, we have $D\left(1+h C^{\prime}(T)\right)^{-[t / h]} \rightarrow D e^{-C^{\prime}(T) t}$. We will show that this estimate also holds in the limit, for the motions $\left(E^{1}\right)^{*}$ and $\left(E^{2}\right)_{*}$ obtained along the same subsequence $\left(h_{k}\right)_{k \geqslant 1}$ (which we will still denote by $(h)_{h>0}$ ).

Fix $\delta<D e^{-C^{\prime}(T) T}$. If $h$ is small enough, we have $\delta \leqslant \operatorname{dist}_{\phi}\left(E_{h}^{1}(t), \Omega \backslash E_{h}^{2}(t)\right)$ for any $t \in[0, T)$. Given a fixed $t<T$, choose a subsequence $\left(h_{k}\right)$ such that both Hausdorff limits of $E_{h_{k}}^{1}(t)$ and $\Omega \backslash E_{h_{k}}^{2}(t)$ exist in $\bar{\Omega}$, and denote them by $K$ and $L$, respectively. Since $\operatorname{dist}_{\phi}\left(E_{h}^{1}(t), \Omega \backslash E_{h}^{2}(t)\right) \geqslant \delta$, in the limit we find $\operatorname{dist}_{\phi}(K, L) \geqslant \delta$. We also have $K \subseteq$ $\left(E^{1}\right)^{*}(t)$ and $L \subseteq \bar{\Omega} \backslash\left(E^{2}\right)_{*}(t)$. Define now $K^{\delta / 2}=K+W_{\phi}(0, \delta / 2)$, which has its boundary between $\partial K$ and $\partial L$ and lies at distance at least $\delta / 2$ from both boundaries. Let $\delta^{\prime}<\delta$ and set $\delta^{\prime \prime}=\left(\delta+\delta^{\prime}\right) / 2$. If $x \in \partial K^{\delta / 2}$, then $W_{\phi}\left(x, \delta^{\prime \prime} / 2\right) \Subset \bar{\Omega} \backslash(K \cup L)$ so that if $h_{k}$ is small enough, then $W_{\phi}\left(x, \delta^{\prime \prime} / 2\right) \subset E_{h_{k}}^{2}(t)$ and $W_{\phi}\left(x, \delta^{\prime \prime} / 2\right) \cap E_{h_{k}}^{1}(t)=\varnothing$. By Lemma 4.2 there exists $\tau>0$, depending only on $\delta^{\prime \prime}$ and $\delta^{\prime}<\delta^{\prime \prime}$, such that $W_{\phi}\left(x, \delta^{\prime} / 2\right) \subset E_{h_{k}}^{2}(s)$ and $W_{\phi}\left(x, \delta^{\prime} / 2\right) \cap E_{h_{k}}^{1}(s)=\emptyset$ for all $t \leqslant s<t+\tau$. In the limit, this implies that for $t \leqslant s<t+\tau$, 
$W_{\phi}\left(x, \delta^{\prime} / 2\right) \subset\left(E^{2}\right)_{*}(s)$ and $\left(E^{1}\right)^{*}(s) \cap W_{\phi}\left(x, \delta^{\prime} / 2\right)=\emptyset$. Since $x$ is an arbitrary point of $\partial K^{\delta / 2}$, this implies that both $\operatorname{dist}_{\phi}\left(\partial K^{\delta / 2}, \partial\left(E^{1}\right)^{*}(s)\right) \geqslant \delta^{\prime} / 2$ and $\operatorname{dist}_{\phi}\left(\partial K^{\delta / 2}, \partial\left(E^{2}\right)_{*}(s)\right) \geqslant$ $\delta^{\prime} / 2$. We deduce that for any $s$ in $(t, t+\tau)$, $\operatorname{dist}_{\phi}\left(\partial\left(E^{1}\right)^{*}(s), \partial\left(E^{2}\right)_{*}(s)\right) \geqslant \delta^{\prime}$. Since $t$ is arbitrary in $[0, T)$ and $\tau$ does not depend on $t$, we deduce that in fact for any $t \in[0, T)$, $\operatorname{dist}_{\phi}\left(\partial\left(E^{1}\right)^{*}(t), \partial\left(E^{2}\right)_{*}(t)\right) \geqslant \delta^{\prime}$. (The case $t=0$ follows directly from Proposition 4.3 .) We may let $\delta^{\prime} \rightarrow \delta$ to see that the inequality holds with $\delta$ instead of $\delta^{\prime}$. In fact, we can deduce from the previous argument that the distance between the two sets decreases at most like $D e^{-C^{\prime}(T) t}$, $t \in[0, T)$.

We have obtained the following result.

Proposition 4.11 Let $E^{1} \subset E^{2} \subset \mathbb{R}^{N}$ be two closed sets with nonempty compact boundary, and assume $\operatorname{dist}_{\phi}\left(\partial E^{1}, \partial E^{2}\right)>0$. Denote by $E_{h}^{1}$ and $E_{h}^{2}, h>0$, the corresponding discrete evolutions in $\mathbb{R}^{N} \times[0,+\infty)$. Let $\left(h_{k}\right)$ be a subsequence such that $E_{h_{k}}^{1} \rightarrow\left(E^{1}\right)^{*}$ and ${ }^{c} E_{h_{k}}^{2} \rightarrow{ }^{c}\left(E^{2}\right)_{*}$ in the Hausdorff sense. Assume also that $E_{h_{k}}^{2} \rightarrow\left(E^{2}\right)^{*}$. Then $\left(E^{1}\right)^{*}(t) \subseteq\left(E^{2}\right)_{*}(t)$ for any $t \geqslant 0$.

In particular, for any $t \in[0, T)$ with $T:=\max \left\{T_{E^{1}}^{*}, T_{E^{2}}^{*}\right\}$, and any $t^{\prime}$ with $t<t^{\prime}<T$,

$$
\operatorname{dist}_{\phi}\left(\partial\left(E^{1}\right)^{*}(t), \partial\left(E^{2}\right)_{*}(t)\right) \geqslant \operatorname{dist}_{\phi}\left(\partial E^{1}, \partial E^{2}\right) e^{-C^{\prime}\left(t^{\prime}\right) t}>0
$$

where $C^{\prime}$ is proportional to the constant in (18).

REMARK 4.12 Notice that the inclusions $\left(E^{1}\right)^{*}(t) \subseteq\left(E^{2}\right)^{*}(t)$ and $\left(E^{1}\right)_{*}(t) \subseteq\left(E^{2}\right)_{*}(t)$ always hold (without any assumption on $\operatorname{dist}_{\phi}\left(\partial E^{1}, \partial E^{2}\right)$ ).

REMARK 4.13 We remark that we do not know if the conclusion of Proposition 4.11 still holds if $\left(E^{1}\right)^{*}$ and $\left(E^{2}\right)^{*}$ are limits of $E_{h_{k}}^{1}$ and $E_{h_{j}}^{2}$ (respectively) along different subsequences. This would be an important result, yielding for instance the uniqueness of the level-set solution $u(x, t)$, defined in Section 4.3.

\subsection{The level-set approach}

Consider now a function $u_{0} \in B U C\left(\mathbb{R}^{N}\right)$ such that for each $t \in \mathbb{R}$ the level-set $\partial\left\{u_{0}>t\right\}$ is bounded. For all $q \in \mathbb{Q}$ consider the level-sets $E^{q}:=\left\{u_{0} \geqslant q\right\}$ and let $E_{h}^{q} \subset \mathbb{R}^{N} \times[0,+\infty)$ be the discrete evolutions of $E^{q}$. Then a diagonal argument shows that, along a subsequence $\left(h_{k}\right)_{k} \geqslant 1$, we have $E_{h_{k}}^{q} \rightarrow\left(E^{q}\right)^{*}$ and ${ }^{c} E_{h_{k}}^{q} \rightarrow{ }^{c}\left(E^{q}\right)_{*}$ locally in the Hausdorff sense, i.e. the distance functions $\operatorname{dist}\left(\cdot, E_{h_{k}}^{q}\right)$ and $\operatorname{dist}\left(\cdot, \mathbb{R}^{N} \times[0,+\infty) \backslash E_{h_{k}}^{q}\right)$ converge to $\operatorname{dist}\left(\cdot,\left(E^{q}\right)^{*}\right)$ and $\operatorname{dist}\left(\cdot, \mathbb{R}^{N} \times[0,+\infty) \backslash\right.$ $\left.\left(E^{q}\right)_{*}\right)$ respectively, uniformly in $\mathbb{R}^{N} \times[0, T]$ for any $T>0$.

Observe that (Remark 4.12) for all $q, r \in \mathbb{Q}$ with $q \geqslant r$, we have $\left(E^{q}\right)^{*}(t) \subseteq\left(E^{r}\right)^{*}(t)$ and $\left(E^{q}\right)_{*}(t) \subseteq\left(E^{r}\right)_{*}(t)$ for any $t \geqslant 0$. Hence we can define two functions $\left.u^{*}, u_{*}: \mathbb{R}^{N} \times 0,+\infty\right) \rightarrow \mathbb{R}$ by letting

$$
u^{*}(x, t):=\sup \left\{q \in \mathbb{Q}: x \in\left(E^{q}\right)^{*}(t)\right\}, \quad u_{*}(x, t):=\sup \left\{q \in \mathbb{Q}: x \in\left(E^{q}\right)_{*}(t)\right\} .
$$

By Proposition 4.11. we know that $\left(E^{q}\right)^{*}(t) \subset\left(E^{r}\right)_{*}(t)$ for any $t \geqslant 0$ whenever $q>r$, which implies $u^{*}(x, t)=u_{*}(x, t)$ for any $(x, t) \in \mathbb{R}^{N} \times[0,+\infty)$. Indeed, if $q>u^{*}(x, t)$, then $x \notin$ $\left(E^{q}\right)^{*}(t)$, so that also $x \notin\left(E^{q}\right)_{*}(t)$, hence $u_{*}(x, t) \leqslant u^{*}(x, t)$; on the other hand, if $q>u_{*}(x, t)$, then if $q^{\prime} \in\left(u_{*}(x, t), q\right) \cap \mathbb{Q}$, we have $x \notin\left(E^{q^{\prime}}\right)_{*}(t) \supset\left(E^{q}\right)^{*}(t)$, hence $x \notin\left(E^{q}\right)^{*}(t)$, and we deduce $u^{*}(x, t) \leqslant u_{*}(x, t)$. We simply denote by $u(x, t)$ this common value. 
Let us observe that, for each $t \geqslant 0$, from Proposition 4.11 (more exactly from the estimate (19)) it follows that $u(\cdot, t)$ is uniformly continuous on $\mathbb{R}^{N}$ (with the same modulus of continuity as $u_{0}$ if $C(T)=0$ in (18)). It also follows easily from Propositions 4.3 and 4.4 that if $\left(x_{n}, t_{n}\right) \rightarrow(x, t)$, then $u\left(x_{n}, t_{n}\right) \rightarrow u(x, t)$ : indeed, for instance, one sees that if $u\left(x_{n}, t_{n}\right)<q$ for $n$ large enough, then $x_{n} \notin\left(E^{q}\right)_{*}\left(t_{n}\right)$, hence in the limit $x \notin\left(E^{q}\right)_{*}(t)$ so that $u(x, t) \leqslant q$. This means that $u$ is globally continuous on $\mathbb{R}^{N} \times[0,+\infty)$. In particular, $\left(E^{q}\right)^{*}(t) \subset\left(E^{s}\right)_{*}(t)$ for any $t \geqslant 0$ whenever $q>s$, $q, s \in \mathbb{R}$. We deduce easily that (letting now $\left.\Gamma^{s}(t):=\left(E^{s}\right)^{*}(t) \backslash\left(E^{s}\right)_{*}(t)\right)$

$$
\bigcup_{t \geqslant 0} \Gamma^{s}(t)=\left(E^{s}\right)^{*} \backslash\left(E^{s}\right)_{*} \subseteq\{(x, t): u(x, t)=s\} .
$$

Now, let $N:=\left\{s \in \mathbb{R}:\left|\left\{(x, t) \in \mathbb{R}^{N} \times[0,+\infty): u(x, t)=s\right\}\right|=0\right\}$. The set $N$ is at most countable. If $s \notin N$, then 20 is in fact an equality: one has $\{u>s\}=\left(E^{s}\right)_{*}$ and $\{u \geqslant s\}=\left(E^{s}\right)^{*}$. One can deduce that $\partial E_{h_{k}}^{s}$ converges to $\{u=s\}$ in the local Hausdorff sense. For these values of $s$, the flow defined by our algorithm is a "true" evolution of hypersurfaces. Indeed, at any time $t \geqslant 0$, we can show that $\{u(\cdot, t)=s\}$ has empty interior. Otherwise, there would exist $W_{\phi}(x, \rho) \subseteq$ $\{u(\cdot, t)=s\}$. In particular, if $q>s>q^{\prime}$ with $q, q^{\prime} \in \mathbb{Q}$, we would have $W_{\phi}(x, \rho) \subseteq\left(E^{q^{\prime}}\right)_{*}(t)$ while $W_{\phi}(x, \rho) \cap\left(E^{q}\right)^{*}(t)=\emptyset$. By (14) and [15), it would follow that if $t \leqslant t^{\prime}<t+\tau_{t+1}(\rho / 2)$, then $W_{\phi}(x, \rho / 2) \subset\left(E^{q^{\prime}}\right)_{*}(t)$ and $W_{\phi}(x, \rho / 2) \cap\left(E^{q}\right)^{*}(t)=\emptyset$, which would imply that $\{u=s\}$ has nonempty interior, leading to a contradiction. We cannot prove in general the uniqueness of the flow $\left(E^{s}\right)^{*}$, since it could depend on the subsequence $\left(h_{k}\right)$ along which the first limits have been taken. If on the contrary $s \in N$, then a fattening of the corresponding level-set happens, and we can only deduce the inclusion (20). As in the case of classical level-set solutions, we expect nonuniqueness of the limit flow in this situation (and only then).

If $G(x, t)=\int_{0}^{t} g(x, s) \mathrm{d} s$ with $g$ continuous, then (by Proposition $4.8 u$ is the unique viscosity solution [15] of

$$
\frac{\partial u}{\partial t}=\phi^{\circ}(\nabla u)\left(\operatorname{div} \nabla \phi^{\circ}(\nabla u)+g\right) .
$$

In particular, in this case, the limit $u$ is the same along any subsequence. We can then deduce that $\partial E_{h}^{s}(t) \rightarrow\{u(\cdot, t)=s\}$ as $h \rightarrow 0$, for each level $s \notin N$, or each time $t$ before the moment the level $s \in N$ fattens.

In case $G$ is an arbirary driving term satisfying (18), we conjecture that our $u$ is still the viscosity solution of

$$
\frac{\partial u}{\partial t}=\phi^{\circ}(\nabla u)\left(\operatorname{div} \nabla \phi^{\circ}(\nabla u)+\frac{\partial G}{\partial t}\right)
$$

built by Lions and Souganidis [23, 24]. However, to show this, we would need either to show the stability of our construction under small perturbations of $G$ (that would allow us to approximate $G$ with smooth functions), or a comparison result like Theorem 2.4 between barriers and viscosity solutions (in the sense of [23, 24]) and then use Corollary 4.6

Let us finally make a few remarks. We first observe that our construction can still be performed if $\phi$ and $\phi^{\circ}$ are nonsmooth: typically, in the crystalline case, where the Wulff shape $\{\phi \leqslant 1\}$ is a polyhedron. In this case, our proof of consistency does not hold (nor is it clear how to extend the definition of a sub/superflow). On the other hand, most of the results are still valid, including the comparison principle in Proposition 4.11, and the construction of the level-set function $u$ starting from $u_{0}$ still makes sense. 
We also mention that in the convex case, if $G=G(t)$, by the same arguments as in [11] we can show that the evolution (defined in $\mathbb{R}^{N}$ ) remains convex for all time, including when the anisotropy is nonsmooth. We also expect that the results in [4] still hold with similar proofs, and that a unique "regular" evolution can be defined for small times as the unique limit of our algorithm, when the initial convex set satisfies an interior $\varepsilon W_{\phi}$-condition. This would in turn yield the uniqueness of the level-set function defined above.

\section{REFERENCES}

1. Almgren, F., TAylor, J. E., \& WAng, L.-H. Curvature-driven flows: a variational approach. SIAM J. Control Optim. 31 (1993), 387-438. Zbl 0783.35002 MR 1205983

2. Ambrosio, L. Movimenti minimizzanti. Rend. Accad. Naz. Sci. XL Mem. Mat. Appl. (5) 19 (1995), 191-246.

3. Ambrosio, L., Fusco, N., \& Pallara, D. Functions of Bounded Variation and Free Discontinuity Problems. Oxford Math. Monogr., Clarendon Press, New York (2000). Zbl 0957.49001 MR 1857292

4. Bellettini, G., Caselles, V., Chambolle, A., \& Novaga, M. Crystalline mean curvature flow of convex sets. Arch. Ration. Mech. Anal. 179 (2006), 109-152. Zbl pre05000053| MR 2208291

5. Bellettini, G., \& Novaga, M. Minimal barriers for geometric evolutions. J. Differential Equations 139 (1997), 76-103. Zbl 0882.35028 MR 1467354

6. Bellettini, G., \& Novaga, M. Comparison results between minimal barriers and viscosity solutions for geometric evolutions. Ann. Scuola Norm. Sup. Pisa Cl. Sci. (4) 26 (1998), 97-131. Zbl 0904.35041 MR 1632984

7. Bellettini, G., \& Novaga, M. Some aspects of De Giorgi's barriers for geometric evolutions. Calculus of Variations and Partial Differential Equations (Pisa, 1996), Springer, Berlin (2000), 115-151. Zbl 0957.35080 MR 1757698

8. Bellettini, G., \& Paolini, M. Some results on minimal barriers in the sense of De Giorgi applied to driven motion by mean curvature. Rend. Accad. Naz. Sci. XL Mem. Mat. Appl. (5) 19 (1995), 43-67; Errata, ibid. 26 (2002), 161-165. Zbl 0944.53039 MR 1387549

9. Bellettini, G., \& Paolini, M. Anisotropic motion by mean curvature in the context of Finsler geometry. Hokkaido Math. J. 25 (1996), 537-566. Zbl 0873.53011 MR 1416006

10. Brakke, K. A. The Motion of a Surface by its Mean Curvature. Math. Notes 20, Princeton Univ. Press, Princeton, NJ (1978). Zbl 0386.53047 MR 0485012

11. Caselles, V., \& Chambolle, A. Anisotropic curvature-driven flow of convex sets. Nonlinear Anal. 65 (2006), 1547-1577. Zbl 1107.35069 MR 2248685

12. Снambolle, A. An algorithm for mean curvature motion. Interfaces Free Bound. 6 (2004), 195-218. Zbl 1061.35147 MR 2079603

13. Chambolle, A., \& Novaga, M. Approximation of the anisotropic mean curvature flow. Math. Models Methods Appl. Sci. 17 (2007), 833-844. Zbl 1120.35054 MR 2334546

14. CHEN, X. Generation and propagation of interfaces for reaction-diffusion equations. J. Differential Equations 96 (1992), 116-141. Zbl 0765.35024 MR 1153311

15. Chen, Y. G., Giga, Y., \& Goto, S. Uniqueness and existence of viscosity solutions of generalized mean curvature flow equations. Proc. Japan Acad. Ser. A Math. Sci. 65 (1989), 207-210. Zbl 0735.35082 MR 1030181

16. De Giorgi, E. New ideas in calculus of variations and geometric measure theory. Motion by Mean Curvature and Related Topics (Trento, 1992), de Gruyter, Berlin (1994), 63-69. Zbl 0808.35004 MR 1277391 
17. Dirr, N., Luckhaus, S., \& Novaga, M. A stochastic selection principle in case of fattening for curvature flow. Calc. Var. Partial Differential Equations 4 (2001), 405-425. Zbl 1015.60070 MR 1867935

18. Evans, L. C., \& Spruck, J. Motion of level sets by mean curvature. I. J. Differential Geom. 33 (1991), 635-681. Zbl 0726.53029 MR 1100206

19. Evans, L. C., \& Spruck, J. Motion of level sets by mean curvature. II. Trans. Amer. Math. Soc. 330 (1992), 321-332. Zbl 0776.53005 MR 1068927

20. Giusti, E. Minimal Surfaces and Functions of Bounded Variation. Birkhäuser, Basel (1984). Zbl 0545.49018 MR 0775682

21. Ilmanen, T. Convergence of the Allen-Cahn equation to Brakke's motion by mean curvature. J. Differential Geom. 38 (1993), 417-461. Zbl 0784.53035 MR 1237490

22. Ilmanen, T. The level-set flow on a manifold. Differential Geometry: Partial Differential Equations on Manifolds (Los Angeles, CA, 1990), Proc. Sympos. Pure Math. 54, Amer. Math. Soc., Providence, RI (1993), 193-204. Zbl 0827.53014 MR 1216585

23. Lions, P.-L., \& Souganidis, P. Fully nonlinear stochastic partial differential equations. C. R. Acad. Sci. Paris Sér. I Math. 9 (1998), 1085-1092. Zbl 1002.60552 MR 1647162

24. Lions, P.-L., \& SougAnidis, P. Fully nonlinear stochastic pde with semilinear stochastic dependence. C. R. Acad. Sci. Paris Sér. I Math. 331 (2000), 617-624. Zbl 0966.60058 MR 1799099

25. Luckhaus, S., \& Sturzenhecker, T. Implicit time discretization for the mean curvature flow equation. Calc. Var. Partial Differential Equations 3 (1995), 253-271. Zbl 0821.35003 MR 1386964

26. YIP, N. K. Stochastic motion by mean curvature. Arch. Ration. Mech. Anal. 144 (1998), 313-355. Zbl 0930.60047 MR 1656479

27. YIP, N. K. Stochastic curvature driven flows. Stochastic Partial Differential Equations and Applications (Trento, 2002), Lecture Notes in Pure Appl. Math. 227, Dekker, New York (2002), 443-460. Zbl 0998.60096 MR 1919523 